\title{
1 Mitochondrial ferritin deficiency reduces male fertility in mice
}

2

3 Running title:

$4 \quad$ Mitochondrial ferritin and male fertility

5

6 Authors.

7 Federica Maccarinelli ${ }^{1}$, Maria Regoni ${ }^{1}$, Fernando Carmona ${ }^{1}$, Esther G. Meyron-

8 Holtzand $^{2}$, Paolo Arosio ${ }^{1 *}$

Affiliations

$12{ }^{1}$ Molecular Biology laboratory, Department of Molecular and Translational Medicine

13 DMMT, University of Brescia, Viale Europa 11, 25123 Brescia, Italy.

14

15

${ }^{2}$ Laboratory for Molecular Nutrition, Faculty of Biotechnology and Food Engineering,

16 Technion-Israel Institute of Technology, Technion City, 32000 Haifa, Israel

${ }^{*}$ Corresponding author

Tel +39 0303717303 ;

Fax +39030 3717305

Email: paolo.arosio@unibs.it

\section{Keywords}

26 Mitochondrial ferritin, spermatogenesis, ATP, sperm motility 


\section{Abstract}

3 Mitochondrial ferritin (FtMt) is a functional ferritin targeted to mitochondria that is 4 highly expressed in the testis. To investigate the role of FtMt in the testis, we set up 5 a series of controlled mating between mice deleted of the FtMt gene $\left(\mathrm{FtMt}^{-1-}\right)$ with $6 \mathrm{FtMt}^{+/+}$mice. We found that the number of newborns per litter and the fertility rate 7 were strongly reduced for the $\mathrm{FtMt}^{-/-}$males, but not for the females, indicating that 8 FtMt has an important role for male fertility. The morphology of the testes and of 9 the spermatozoa of $\mathrm{FtMt}^{-1-}$ was normal, and we did not detect alterations in sperm 10 parameters and increase of oxidative stress indices. In contrast we observed that 11 cauda epididymis of $\mathrm{FtMt}^{-/}$mice were significantly lighter and contained less 12 spermatozoa than those of controls, and that the ATP content of the $\mathrm{FtMt}^{-1 /}$ sperm 13 was lower than that of the $\mathrm{FtMt}^{+/+}$one. We concluded that FtMt contributes to 14 spermatogenesis and to male fertility. 


\section{Introduction}

2

Mitochondrial ferritin (FtMt) is a functional ferritin targeted to mitochondria that is highly expressed in testes of many species, such as human, mouse and drosophila (Levi and Arosio 2004), where protects from iron-induced damage through the regulation of mitochondrial iron availability (Campanella, et al 2009). Mitochondria play an important role in spermatogenesis, as indicated by the findings that in drosophila defects in mitochondrial fusion processes (Hales and Fuller 1997) or mutations of the spermatogenesis-specific cytochrome gene cyt-c-d (Arama, et al 2006) lead to male sterility. In addition, the finding that the inactivation in drosophila of the dmfrn gene, which encodes the homolog of human mitoferrin2 responsible for mitochondrial iron incorporation, caused a male sterility and was rescued by a low iron diet (Metzendorf and Lind 2010) indicated that mitochondrial iron metabolism has an essential role in spermatogenesis, The testes are very rich in mitochondria and express high levels of proteins involved in mitochondrial iron transport and metabolism, such as frataxin and mitoferritin2. Testicular transferrin is the major secretory product of Sertoli cells with a critical role in the iron delivery to the germinal cells (Skinner and Griswold 1980) and transferrin receptor was detected mainly on early spermatocytes, supporting a need for these cells to take up iron (Leichtmann-Bardoogo, et al 2012). In addition, seminal transferrin concentration was found to be correlated with the sperm count in human sperm (Orlando, et al 1985) and severe iron overload, as it occurs in homozygous $\beta$ thalassemia patients, leads to oxidative damage and reduces male fertility (Perera, et al 2002). Thus, abnormalities of the expression of iron metabolism proteins in the testes and particularly in the spermatozoa may contribute to male infertility. A previous communication indicated that FtMt is highly expressed in the human sperm and that its concentration is significantly reduced in asthenospermic samples (Calzi, et al 2003). Moreover, a proteomic study of the sperm of fertile and unfertile subjects aimed to identify protein biomarkers, found FtMt to be the most prominent protein of interest since it was the only one among the 128 proteins 
1 identified that was present in the control sperm samples but totally absent in all the

2 abnormal sperms (Behrouzi, et al 2013). This stimulated the interest in proteins involved in mitochondrial iron metabolism, particularly FtMt, with regard to male

4 infertility. Mouse strains deficient in the FtMt have been described (Bartnikas, et al

5 2010, Maccarinelli, et al 2014) and they were found to have normal hematological

6 indices (Bartnikas, et al 2010) and to be more sensitive to the cardiotoxic drug

7 doxorubicin (Maccarinelli, et al 2014). After the testis, the heart is one of the organs

8 richest in FtMt and the finding that its absence makes the heart more sensitive to

9 oxidative damage, supports the hypothesis that FtMt has a cytoprotective activity

10 (Arosio and Levi 2010). FtMt-null mice are fertile, but the role of FtMt in the testis,

11 where its concentration is the highest, remains unexplored. In this work we

12 analyzed these mice in more detail, showing a significant decrease in male fertility

13 that was not apparently associated to defect in sperm motility but to a lower level of 14 ATP.

\section{Materials and Methods}

Animals. All mice analyzed were 3-month-old in the C57BL/6J background. All the procedures followed animal protection laws and institutional guidelines of the European Convention for the Protection of Laboratory Animals. The study was approved by the Institutional Animal Care and Use Committee of the University of Brescia and the Italian Ministry of Science and Research. Breeding study. Every male mouse $\left(n=8 \mathrm{FtMt}^{-/-} ; n=8 \mathrm{FtMt}^{+/+}\right.$) was housed with two virgin $\mathrm{FtMt}^{+/+}$females and, in parallel, $10 \mathrm{FtMt}^{-/}$and $4 \mathrm{FtMt}^{+/+}$male mice were 25 housed each with two virgin $\mathrm{FtMt}^{-/-}$females. After two weeks, all mice were housed separately for other 4 weeks during which we evaluated the pregnancy rate expressed as a percentage of pregnant females in respect to the total number of mating females (fertility rate), and the number of pups per litter (litter size). 
1 Testes and sperm collection. Mice were anesthetized with Avertine $(23 \mu \mathrm{L} / \mathrm{g}$ mouse; Sigma) and perfused with a physiological saline solution containing $2 \%$ heparin. The testes were removed and fixed in Bouin's Solution (Sigma) for 3 hours, gradually dehydrated and embedded in paraffin. The cauda epididymis were removed, weighted and placed in $500 \mu \mathrm{L}$ of Dulbecco's modified Eagle's medium (D-MEM Life Technologies; 2\% Fetal Bovine Serum, Sigma; $0.45 \%$ glucose; $0.6 \%$ HEPES) for sperm isolation. The medium was pre-warmed at $37^{\circ} \mathrm{C}, 5 \% \mathrm{CO}_{2}$ for 2 h. Each cauda was punctured several times with a 26G needle and the spermatozoa were allowed to swim out with the help of gentle squeezing with surgical scissors. Spermatozoa were allowed to disperse in the medium for $60 \mathrm{~min}$ at $37^{\circ} \mathrm{C}, 5 \% \mathrm{CO}_{2}$ with gentle agitation every $10 \mathrm{~min}$ and then transferred to micro centrifuge tubes for collection and analysis.

Sperm parameters analysis: concentration, morphology and motility. Sperm concentration of $14 \mathrm{FtMt}^{-/-}$mice and 9 controls was determined by counting in a Burker's chamber $20 \mu \mathrm{L}$ of the sperm isolate diluted 10 -fold. For morphological analysis $2 \times 10^{6}$ spermatozoa $\left(n=3 \mathrm{FtMt}^{-/} ; \mathrm{n}=3 \mathrm{FtMt}^{+/+}\right.$) were re-suspended in $4 \%$ paraformaldehyde (PFA) and incubated at $4^{\circ} \mathrm{C}$ for $30 \mathrm{~min}$. Fixed spermatozoa were mounted on polarized slides, counterstained with Hematoxylin for 5 min and observed by optical microscopy using 20X and 40X magnification. Moreover, two $\mathrm{FtMt}^{-{ }^{-}}$mice were sent to the Embryology Laboratory of Charles River (Lyon, France) where the sperm was analyzed with Computer Assisted Sperm Analysis tool (CASA) to detect cells number and motion.

Mitochondrial staining. Sperm isolate was re-suspended $10^{4}$ cells $/ \mu \mathrm{L}$ in $200 \mu \mathrm{L}$ of $250 \mathrm{nM}$ MitoTracker Red (Invitrogen) and incubated at $37^{\circ} \mathrm{C}$ for $30 \mathrm{~min}$ to stain live mitochondria. Then, samples were fixed in 4\% PFA, mounted and incubated with DAPI $0.1 \mu \mathrm{g} / \mathrm{mL}$ for $15 \mathrm{~min}$ to counterstain the nucleus. The slides were observed using standard fluorescence microscopy.

ATP assay. ATP concentration in sperm was measured using ATP luminescence assay kit (CellTiter-Glo ${ }^{\circledR}$ Luminescent Cell Viability Assay, Promega), according to manufacturer's instructions. Sperm isolate $\left(n=3 \quad \mathrm{FtMt}^{-1} ; n=3 \quad \mathrm{FtMt}^{+/+}\right)$was re- 
suspended 400 cells $/ \mu \mathrm{L}$ in $1 \mathrm{~mL}$ of D-MEM. Luminescence of $100 \mu \mathrm{L}$ of sample was measured with the EnSight Multimode Plate Reader (PerkinElmer). ATP luminescence was normalized to the concentration of the protein extracts.

Histology of testis and of epididymis. Paraffin embedded testes of 12-week-old mice $\left(n=3 \mathrm{FtMt}^{-/} ; n=3 \mathrm{FtMt}^{+/+}\right)$were sectioned and the $4 \mu \mathrm{m}$ thick slices then stained with Hematoxylin for 1 min.

Western Blots. Sperm isolate $\left(n=3 \mathrm{FtMt}^{--} ; n=3 \mathrm{FtMt}^{+/+}\right)$was centrifuged and lysed in $100 \mu \mathrm{L}$ of homogenization lysis buffer with protease inhibitors (Complete Protease Inhibitor Cocktail; Roche: 200 mM Tris- $\mathrm{HCl}$ pH 8, 100 mM NaCl, 1 mM EDTA, $0.5 \%$ NP-40, $10 \%$ glycerol, $1 \mathrm{mM}$ sodium orthovanadate, $1 \mathrm{mM}$ sodium fluoride). Protein concentration was measured by Bradford assay. The FtMt was detected by western blotting: $20 \mu \mathrm{g}$ of soluble proteins were heated at $70^{\circ} \mathrm{C}$ for $10 \mathrm{~min}$, centrifuged at $13,000 \mathrm{rpm}$ at $4^{\circ} \mathrm{C}$ and the supernatants were loaded on $8 \%$ denaturing-PAGE. For detection, a rabbit antibody for mouse FtMt was used (Santambrogio, et al 2007). Band intensity was revealed by enhanced chemiluminescence (ECL; GE Healthcare) and visualized with the Kodak Image Station 440CF (Kodak).

SOD Activity. SOD analysis was performed separating $20 \mu \mathrm{g}$ of sperm protein extracts on $12 \%$ non-denaturing PAGE. Protein level in? the gel was blotted and probed with rabbit anti-MnSOD antibody (Merk Millipore). For SOD activity (Cavadini, et al 2007) the polyacrylamide gel was incubated at room temperature in the dark with $50 \mathrm{~mL}$ of $50 \mathrm{mM}$ phosphate, $0.2 \mathrm{mM} \mathrm{NBT}, 0.03 \mathrm{mM}$ riboflavin and 0.1 $\mathrm{mM}$ EDTA for $15 \mathrm{~min}$. After adding tetramethylethylenediamine (TEMED) to $15 \mathrm{mM}$ final concentration, the gel was incubated for another $20 \mathrm{~min}$ at room temperature in presence of light. 


\section{Results}

Male and female $\mathrm{FtMt}^{-/-}$mice are viable and do not show an evident phenotype.

3 They are fertile, but we found some difficulties in maintaining a homozygous $\mathrm{FtMt}^{-1}$

4 colony, since the number of pups per litter and the number of litters were generally

5 very low. Thus, we set up a series of controlled mating between $\mathrm{FtMt}^{+/+}$and $\mathrm{FtMt}^{-/-}$

6 mice to verify whether the fertility problems were associated with the females or the

7 males. Each male was mated with two virgin females for two weeks and then each mouse housed separately for other 4 weeks during which we evaluated the

9 percentage of pregnant females in respect to the total number of mating females

10 (fertility rate), and the number of pups per litter (litter size). The experiment

11 consisted in the crossing for two consecutive weeks of $8 \mathrm{FtMt}^{-/}$and $8 \mathrm{FtMt}^{+/+}$males

12 with two $\mathrm{FtMt}^{+/+}$females/each, and $10 \mathrm{FtMt}^{-/}$and $4 \mathrm{FtMt}^{+/+}$males with two $\mathrm{FtMt}^{-1-}$

13 females/each and in analyzing the newborns. The results showed that the males

14 with the $\mathrm{FtMt}^{-/}$genotype had a mean number of pups per litter that was about half

15 of that of males with the $\mathrm{FtMt}^{+/+}$genotype, irrespective of the female genotype, 16 while no differences were observed between $\mathrm{FtMt}^{-{ }_{-}-}$and $\mathrm{FtMt}^{+/+}$females (Fig. 1A).

17 Furthermore, the fertility rate of the crossings with $\mathrm{FtMt}^{-/ 2}$ males was significantly 18 lower compared to that obtained crossing $\mathrm{FtMt}^{+/+}$males with $\mathrm{FtMt}^{-/-}$females (Fig.

19 1B). We concluded that the deletion of the FtMt gene impaired significantly the male fertility but not the female fertility, in keeping with the observation that FtMt is highly expressed in testis, particularly in the spermatozoa.

Next we performed histological analyses of Bouin's fixed testis sections from $\mathrm{FtMt}^{-1-}$ and $\mathrm{FtMt}^{+/+}$mice. No evident morphological abnormalities were found (Fig. 2A) and the weight of the testis and the number of spermatocytes were apparently normal in the presence or absence of FtMt. In contrast the cauda epydidymis of $\mathrm{FtMt}^{-/}$ mice were significantly lighter and contained a lower number of spermatozoa than those of $\mathrm{FtMt}^{+/+}$mice (Fig. 2B and 2C). For sperm analysis, spermatozoa were collected from the cauda epididymis, counted and analyzed by light microscopy before and after hematoxylin staining. We could not detect any evident difference in the number and the morphology of the FtMt-null spermatozoa and also after 
staining with MitoTracker no differences were observed between the two murine strains. A more detailed analysis of sperm is normally obtained with automated capture video system using a Computer Assisted Sperm Analysis system (CASA), a technique not available near our laboratory. For this reason, we sent two $\mathrm{FtMt}^{-1-}$ mice to the Embryology Laboratory of Charles River (Lyon, France). Compared with the in-house controls, no significant alterations were found in the total number of spermatozoa or in the percentage of spermatozoa with progressive motility (data not shown). A greater number of bent-head spermatozoa was observed in the $\mathrm{FtMt}^{-1-}$ mice (data not shown), but it was not statistically significant.

Finally, we performed biochemical analyses. Western blotting of the spermatozoa extracts confirmed the absence of FtMt in the $\mathrm{FtMt}^{-/}$mice, but no evident differences were detected in the level of mitochondrial manganese SOD2 protein (Fig. 3A) and SOD activity (not shown), confirming an equal number of mitochondria in the $\mathrm{FtMt}^{-/}$and $\mathrm{FtMt}^{+/+}$spermatozoa and a normal oxidative stress status. In contrast, the measured ATP concentration of freshly isolated spermatozoa was significantly reduced in the $\mathrm{FtMt}^{-/-}$mice (Fig. 3B).

\section{Discussion}

It was shown that FtMt has a cytoprotective activity in human cells in culture (Arosio and Levi 2010, Campanella, et al 2009) and that its presence protects mice from doxorubicin cardiotoxicity (Maccarinelli, et al 2014). Thus, it was expected that its absence could affect also the functionality of the testis, the organ with the highest expression of FtMt (Santambrogio, et al 2007). Surprisingly an initial report of $\mathrm{FtMt}^{-1-}$ mice showed no evidence for impaired fertility (Bartnikas, et al 2010), but the more careful analysis of the present study reveals a lower than normal fertility rate and a decrease to about half in the number of pups per litter from $\mathrm{FtMt}^{-/}$males compared to that from $\mathrm{FtMt}^{+/+}$males. In this study, our analyses of the male reproductive tract and sperm of FtMt-null mice identified a few significant differences compared to the control mice, consisting in a lower weight of the cauda 
epididymis containing fewer spermatozoa and in a decreased sperms ATP level. However, all major parameters of sperm morphology and motility did not show evident differences compared to C57BL reference values, in agreement with the fact that these mice are not infertile, but clearly, the mature sperm was less 5 functional in the $\mathrm{FtMt}^{-/-}$mice. The spermatozoa we analyzed were those that spontaneously moved out from the cauda epididymis, a procedure that selects the fastest from the ones with low motility. Moreover, these cells have to undergo the transport on ductal system for full maturation and acquisition of complete motility. It is conceivable that the lack of FtMt and the reduced level of ATP may have a negative effect on these last steps of maturation resulting in a reduction of their motility and activity. This hypothesis is supported by two recent studies. One of them showing a reduced level of the mitochondrial ferritin in all asthenospermic sperms analyzed (Calzi, et al 2003), and the other showing FtMt to be the only one protein present in the control sperms but totally absent in all the abnormal samples, characterized by a low motility and/or DNA fragmentation (Behrouzi, et al 2013). In conclusion this work supports the hypothesis that FtMt contributes to spermatogenesis and thus to male fertility, with mechanisms that remain to be elucidated that may involve mitochondrial protection from oxidative damage. It also supports the suggestion that FtMt may be used as a marker of sperm motility and quality.

\section{Acknowledgments}

We are grateful to Dr Andreas Meinhardt for precious suggestions and comments and to Dr Marco Spiller and Dr Daria Leali for the support in sperm analysis. The work was partially supported by MIUR grant PRIN10-11 to PA and by Telethon grant GGP1099 to PA

\section{References}

Arama, E., Bader, M., Srivastava, M., Bergmann, A. \& Steller, H. (2006) The two Drosophila cytochrome $\mathrm{C}$ proteins can function in both respiration and caspase activation. Embo j, 25, 232-243. 
Arosio, P. \& Levi, S. (2010) Cytosolic and mitochondrial ferritins in the regulation of cellular iron homeostasis and oxidative damage. Biochim Biophys Acta, 1800, 783-792.

Bartnikas, T.B., Campagna, D.R., Antiochos, B., Mulhern, H., Pondarré, C. \& Fleming, M.D. (2010) Characterization of mitochondrial ferritin-deficient mice. Am J Hematol, 85, 958-960.

Behrouzi, B., Kenigsberg, S., Alladin, N., Swanson, S., Zicherman, J., Hong, S.H., Moskovtsev, S.I. \& Librach, C.L. (2013) Evaluation of potential protein biomarkers in patients with high sperm DNA damage. Syst Biol Reprod Med, 59, 153-163.

Calzi, F., Levi, S., Santambrogio, P., De Santis, L., Rabellotti, E. \& Bonzi, V. (2003) Spermatozoa mitochondrial ferritin (MtF) content is related to sperm motility. Fertility and Sterility, 80, 31.

Campanella, A., Rovelli, E., Santambrogio, P., Cozzi, A., Taroni, F. \& Levi, S. (2009) Mitochondrial ferritin limits oxidative damage regulating mitochondrial iron availability: hypothesis for a protective role in Friedreich ataxia. Hum Mol Genet, 18, 1-11.

Cavadini, P., Biasiotto, G., Poli, M., Levi, S., Verardi, R., Zanella, I., Derosas, M., Ingrassia, R., Corrado, M. \& Arosio, P. (2007) RNA silencing of the mitochondrial ABCB7 transporter in HeLa cells causes an iron-deficient phenotype with mitochondrial iron overload. Blood, 109, 3552-3559.

Hales, K.G. \& Fuller, M.T. (1997) Developmentally regulated mitochondrial fusion mediated by a conserved, novel, predicted GTPase. Cell, 90, 121-129.

Leichtmann-Bardoogo, Y., Cohen, L.A., Weiss, A., Marohn, B., Schubert, S., Meinhardt, A. \& Meyron-Holtz, E.G. (2012) Compartmentalization and regulation of iron metabolism proteins protect male germ cells from iron overload. Am J Physiol Endocrinol Metab, 302, E1519-1530.

Levi, S. \& Arosio, P. (2004) Mitochondrial ferritin. Int J Biochem Cell Biol, 36, 18871889.

Maccarinelli, F., Gammella, E., Asperti, M., Regoni, M., Biasiotto, G., Turco, E., Altruda, F., Lonardi, S., Cornaghi, L., Donetti, E., Recalcati, S., Poli, M., Finazzi, D., Arosio, P. \& Cairo, G. (2014) Mice lacking mitochondrial ferritin are more sensitive to doxorubicin-mediated cardiotoxicity. $J$ Mol Med (Berl), 92, 859-869.

Metzendorf, C. \& Lind, M.I. (2010) Drosophila mitoferrin is essential for male fertility: evidence for a role of mitochondrial iron metabolism during spermatogenesis. BMC Dev Biol, 10, 68.

Orlando, C., Caldini, A.L., Barni, T., Wood, W.G., Strasburger, C.J., Natali, A., Maver, A., Forti, G. \& Serio, M. (1985) Ceruloplasmin and transferrin in human seminal plasma: are they an index of seminiferous tubular function? Fertil Steril, 43, 290-294.

Perera, D., Pizzey, A., Campbell, A., Katz, M., Porter, J., Petrou, M., Irvine, D.S. \& Chatterjee, R. (2002) Sperm DNA damage in potentially fertile homozygous beta-thalassaemia patients with iron overload. Hum Reprod, 17, 1820-1825. 
1 Santambrogio, P., Biasiotto, G., Sanvito, F., Olivieri, S., Arosio, P. \& Levi, S. (2007) Mitochondrial ferritin expression in adult mouse tissues. $J$ Histochem

3 Cytochem, 55, 1129-1137.

4 Skinner, M.K. \& Griswold, M.D. (1980) Sertoli cells synthesize and secrete 5 transferrin-like protein. J Biol Chem, 255, 9523-9525. 

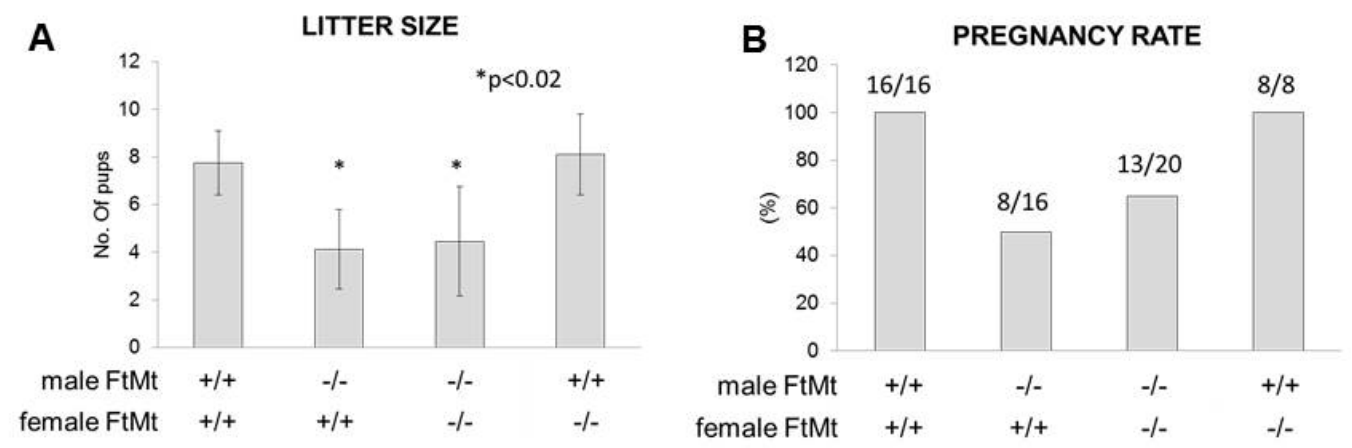

3

4 Figure 1: Breeding study. Each mouse male $\left(\mathrm{n}=8 \mathrm{FtMt}^{-/-} ; \mathrm{n}=8 \mathrm{FtMt}^{+/+}\right)$was 5 housed with two virgin $\mathrm{FtMt}^{+/+}$females and, in parallel, $10 \mathrm{FtMt}^{-/-}$and $4 \mathrm{FtMt}^{+/+}$male 6 mice were housed each with two virgin $\mathrm{FtMt}^{-/-}$females. After two weeks all mice 7 were housed separately for other 4 weeks during which we evaluated the number 8 of pups per litter (litter size) (A) and the fertility rate expressed as percentage of 9 pregnant females respect the total number of mating females (B). The number of 10 pregnant females and of total females analyzed is presented above the columns. 


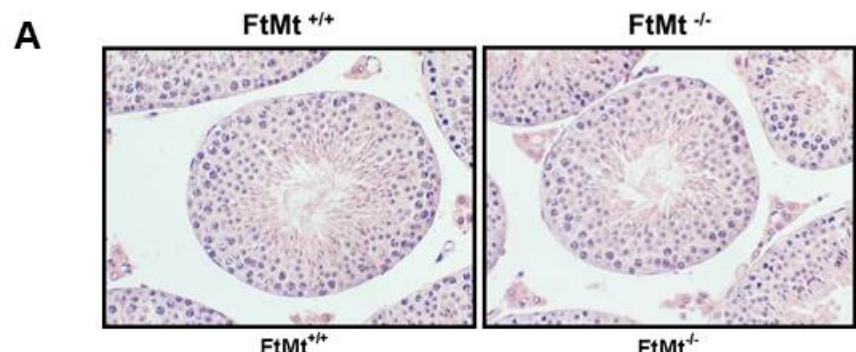

Testis

B

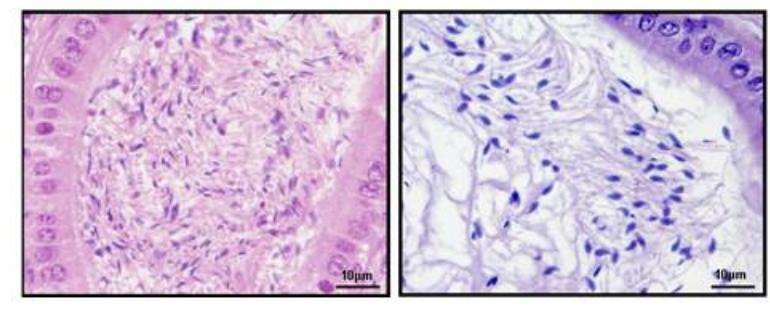

Epididymis

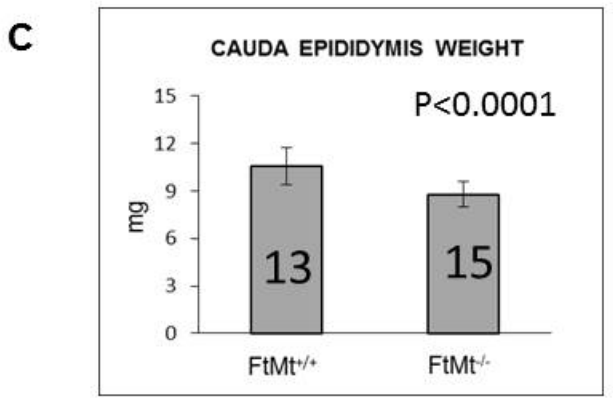

Figure 2

1

3 Figure 2. Morphological analysis of male reproductive tract. (A) Paraffin

4 embedded testes of $\mathrm{FtMt}^{-/-}$and $\mathrm{FtMt}^{+/+}$mice were sectioned and stained with

5 Hematoxylin. No evident difference in morphology or spermatocyte

6 number/maturation is observed. (B) Hematoxylin stain of epididymis sections

7 showed a lower density of spermatozoa in FtMt -/- mice. (C) Before sperm analysis

8 cauda epididymis were collected and weighed. The data from $13 \mathrm{FtMt}^{+/+}$and 15

$9 \mathrm{FtMt}^{-/-}$mice show that the mean cauda epididymis weight of $\mathrm{FtMt}^{-1-}$ mice is 10 significantly lower than those of the controls. 


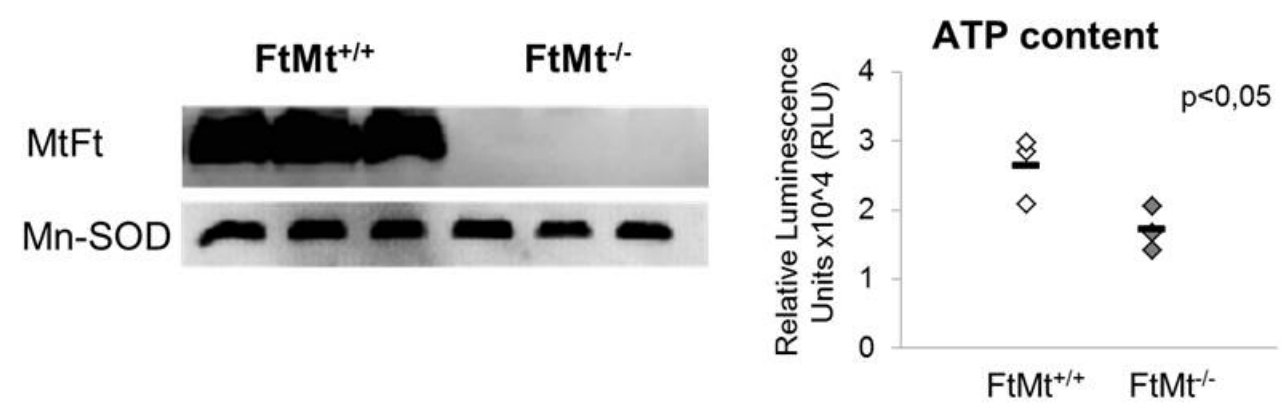

1

2

3 Figure 3: Biochemical analyses. (A) Immunoblotting of $20 \mu \mathrm{g}$ protein extracts 4 from spermatozoa of $\mathrm{FtMt}^{+/+}$and $\mathrm{FtMt}^{-/-}$with anti-FtMt antibody confirmed the 5 absence of the protein in sperm $\mathrm{FtMt}^{-/}$. No differences were revealed in oxidative 6 stress indices analyzed including manganese superoxide dismutase (Mn-SOD). (B) 7 Evaluation of ATP content in the homogenate of freshly isolated sperm from 3 $8 \mathrm{FtMt}^{+/+}$and $3 \mathrm{FtMt}^{-/-}$mice. The luminescence values were normalized to the 9 concentration of the protein extracts obtained from the same volume of sperm 10 isolate. ATP content showed to be significantly decreased in the absence of FtMt. 